\title{
Stabilizer-free poly(lactide-co-glycolide) nanoparticles for multimodal biomedical probes
}

\author{
Fong-Yu Cheng ${ }^{a}$, Saprina Ping-Hsien Wang ${ }^{b}$, Chio-Hao Su ${ }^{\text {a,c }}$, Tsung-Liu Tsai ${ }^{\text {d }}$, Ping-Ching Wu ${ }^{\mathrm{d}}$, \\ Dar-Bin Shieh ${ }^{\mathrm{d}, \mathrm{e}}$, Jyh-Horng Chen ${ }^{\mathrm{c}}$, Patrick Ching-Ho Hsieh ${ }^{\mathrm{b}, * *}$, Chen-Sheng Yeh ${ }^{\mathrm{a}, *}$

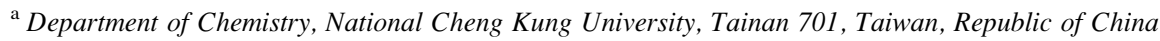 \\ ${ }^{\mathrm{b}}$ Institutes of Clinical Medicine \& Biomedical Engineering and Department of Surgery, National Cheng Kung University, \\ Tainan 704, Taiwan, Republic of China \\ ${ }^{\mathrm{c}}$ Interdisciplinary MRI/MRS Lab, Department of Electrical Engineering, National Taiwan University, Taipei 106, Taiwan, Republic of China \\ ${ }^{\mathrm{d}}$ Institute of Basic Medical Sciences, National Cheng Kung University, Tainan 701, Taiwan, Republic of China \\ ${ }^{\mathrm{e}}$ Institute of Oral Medicine, National Cheng Kung University, Tainan, 701, Taiwan, Republic of China
}

Received 16 November 2007; accepted 18 January 2008

Available online 13 February 2008

\begin{abstract}
Apart from the reported PLGA submicro- and microspheres with broad size distribution, we have successfully developed a methodology using nanoprecipitation to prepare different sizes of PLGA nanoparticles with narrow size distributions. The newly developed PLGA nanoparticles could be readily modified with hydrophilic biomaterials on their surface and entrap hydrophobic drugs into their interiors. The encapsulation of FITC inside PLGA nanoparticles displayed a controlled release of drug system. The surfaces of the FITC entrapped PLGA nanoparticles were conjugated with quantum dots to serve as bimodal imaging probes. For nuclear transport, combination of nuclear localization signal (NLS) and PLGA nanoparticles, PLGA nanoparticles could successfully enter into HeLa cells nuclei. From tissue uptake results, PLGA nanoparticles had more uptaken by brain and liver than other tissues. The iron oxide nanoparticles-conjugated PLGA nanoparticle showed high efficiency of relaxivities $r 2$ and could be used as the powerful magnetic resonance imaging (MRI) agents.
\end{abstract}

(C) 2008 Elsevier Ltd. All rights reserved.

Keywords: PLGA; Nanoparticles; Drug delivery; Nuclear localization signal; MRI

\section{Introduction}

Poly(D,L-lactide-co-glycolide) (PLGA) polymers, approved by the U.S. Food and Drug Administration (FDA), have attracted significant interest for tissue engineering applications and delivery system because they are less toxic, biodegradable and biocompatible [1-5]. More importantly, the by-products of PLGA, the lactic acid and glycolic acid, can be eliminated from the body as carbon dioxide and water through the tricarboxylic acid cycle. Amongst the PLGA applications,

\footnotetext{
* Corresponding author.

** Corresponding author.

E-mail addresses: phsieh@mail.ncku.edu.tw (P.C.-H. Hsieh), csyeh@mail. ncku.edu.tw (C.-S. Yeh).
}

the most interesting one is probably the carrier delivery system that encapsulates drugs and releases them under a controlled mechanism [6-11]. However, it is difficult for large size PLGA microparticles to deliver drugs to target tissues by systemic circulation or across the mucosal membrane. PLGA nanoparticles (PLGA NPs), with their nano-scale size, have been proven to be more efficient than microparticles in transfection and tissue and cell uptakes. Although inorganicbased nanomaterials such as Au, quantum rods, and carbon nanotubes also show promise for biomedical applications, their biocompatibility, cytotoxity and metabolism in living tissues remain largely uncharacterized.

The unique structure of PLGA NPs, composed of a hydrophilic surface and a hydrophobic core, provides a drug carrying reservoir and also enables them to dissolve in 
aqueous solutions. Many approaches are proposed for the preparation of PLGA NPs. The emulsification-evaporation method [3,11-13], spontaneous emulsification-solvent diffusion method (SESD) [14,15], and nanoprecipitation method $[6,16]$ are all widely used for preparing various diameters of PLGA NPs. During nanoparticles formation by emulsification-evaporation and SESD approaches, toxic organic solvents such as $\mathrm{CH}_{2} \mathrm{Cl}_{2}$ and $\mathrm{CHCl}_{3}$ are usually used. To meet the requirement for clinical use, the residual solvents should be completely removed from the PLGA particles.

The aggregation of PLGA NPs during solvent-evaporation process is a notable problem regardless different preparation method. In order to prevent PLGA NP aggregation, polymer stabilizers are often used. Many stabilizers such as poly(vinyl alcohol) (PVA), poly(vinyl pyrrolidone) (PVP), Tween 80, Fluonic 127 (poloxamer 407), Fluonic 68 (poloxamer 188), didodecyl dimethyl ammonium bromide (DMAB) are also excellent stabilizer candidates [2,6,8-12,14-18]. These stabilizers are coated on the surface of PLGA NPs and can affect the zeta potential, particle size and particle surface properties. However, although polymer stabilizers may prevent nanoparticles aggregation, they are difficult to be removed even through thorough washing. Furthermore, most polymer stabilizers do not have functional groups for further modifications, which significantly limit their biomedical applications. Until now we have only a few studies demonstrated successful biomolecule conjugation using stabilizers and this process usually requires extended experiment time such as $24 \mathrm{~h}$ for reaction [19].

Uniform and small particle size of nanoparticles is an important factor for cellular uptake and tissue targeting. It has been reported that the size of PLGA NPs ranged mainly from 100 to $500 \mathrm{~nm}$ and the standard deviation was easily up to $30 \%$ or more. Traditionally the size of PLGA NPs was measured using photon correlation spectroscopy (PCS, also called dynamic light scattering). However, PCS measurement may not as precisely as the measurement using transmission electronic microscopy (TEM) which reflect the exact particle size whereas PCS measures the hydrodynamic diameter of PLGA NPs. For examples, Carlos et al. reported $38.6 \pm$ $0.2 \mathrm{~nm}$ and $67.1 \pm 0.2 \mathrm{~nm}$ PLGA NPs from PCS, but large distribution $(15-40 \%)$ was observed from TEM images [13]. Thirumala et al. reported $20.2 \pm 0.2 \mathrm{~nm}$ and $157 \pm$ $0.9 \mathrm{~nm}$ PLGA NPs using PCS, but their standard deviation was broad (50-70\%) in TEM images [16]. Therefore, how to prepare PLGA NPs in a uniform size is still a challenging task.

\section{Experimental section}

\subsection{Materials}

Copolymers of PLGA (50DGOH-040, M.W. 35,000-65,000 Da and 50DGOH-020, M.W. 16,000-35,000 Da) with the ratio (50/50) of lactide to glycolide were purchased from Bio Invigor Co. Qdot 525 ITK $^{\mathrm{TM}}$ amino (PEG) quantum dots and Qdot 655 ITK $^{\mathrm{TM}}$ amino (PEG) quantum dots (PEG- $\mathrm{NH}_{2}-\mathrm{QD} 525$ and PEG- $\mathrm{NH}_{2}-\mathrm{QD} 655$, respectively) were purchased from Invitrogen, USA. 1-Ethyl-3-(3-dimethylaminopropyl)-carbodiimide
(EDC), fluorescein isothiocyanate (FITC) and succinimidyl-4-[N-maleimidomethyl]-cyclohexane-1-carboxylate (SMCC) were all obtained from Sigma Chemical $\mathrm{Co} . \mathrm{Fe}_{3} \mathrm{O}_{4}$ nanoparticles are prepared based on our previous report [20]. All chemicals and reagents were of analytical grade.

\subsection{Preparation of PLGA nanoparticles (PLGA NPS)}

PLGA (50DGOH-040) was dissolved in $5 \mathrm{~mL}$ of acetone (the solvent) at the concentration of $10 \mathrm{mg} / \mathrm{mL}$. The ethanol/ $\mathrm{H}_{2} \mathrm{O}(50 / 50, \% \mathrm{v} / \mathrm{v})$ aqueous (the non-solvent) was added by dropwise $(2 \mathrm{~mL} / \mathrm{min})$ into the PLGA solution using tubing pump and stirred at $400 \mathrm{rpm}$ by a magnetic stirrer until turbidity appeared. Following 5 more minutes of stirring, the suspension was transferred into $20 \mathrm{~mL}$ of deionized water in a glass beaker and stirred at $400 \mathrm{rpm}$ for another $20 \mathrm{~min}$. The organic solvent was then removed under reduced pressure. To exclude aggregation of PLGA, the solution was filtered with $1 \mu \mathrm{m}$ filter paper. The collected suspension contained $72 \pm 6.8 \mathrm{~nm}$ of PLGA NPs solution. The $125.8 \pm 6.8 \mathrm{~nm}$ PLGA NPs were prepared based on the same protocol with $20 \mathrm{mg} / \mathrm{mL}$ PLGA (50DGOH-040) in acetone instead of $20 \mathrm{mg} / \mathrm{mL}$. For the preparation of $192.6 \pm 15.2 \mathrm{~nm}$ PLGA NPs, $10 \mathrm{mg} / \mathrm{mL}$ PLGA (50DGOH-020) was used. The final concentration of the collected PLGA NPs solutions was measured by the following procedure. An eppendorf $(1.5 \mathrm{~mL})$ was weighted and recorded before adding $1 \mathrm{~mL}$ of PLGA NPs solution. The PLGA NPs solution was centrifuged at 13,000 rpm for $10 \mathrm{~min}$ and the supernatant was discarded. The precipitate was washed with $1 \mathrm{~mL}$ deionized water and dried under vacuum for $24 \mathrm{~h}$. The weight of PLGA precipitates was measured.

\subsection{Preparation of FITC-encapsulated PLGA nanoparticles (FITC-PLGA NPS)}

PLGA (50 mg) and $2 \mathrm{mg}$ of FITC (dissolved in DMSO before use) were dissolved in $5 \mathrm{~mL}$ of acetone. FITC was encapsulated using the same protocol for preparing PLGA NPs. An eppendorf $(1.5 \mathrm{~mL})$ tube was weighted and recorded before adding $1 \mathrm{~mL}$ of FITC-PLGA NPs solution. The eppendorf tube containing FITC-PLGA NPs was centrifuged at 13,000 rpm for $10 \mathrm{~min}$ and the supernatant was discarded. The precipitates were washed with $1 \mathrm{~mL}$ deionized water and dried under vacuum for $24 \mathrm{~h}$ before the weight was determined. We then added $300 \mu \mathrm{L}$ of acetone into the eppendorf to completely dissolve the precipitates. The fluorescent intensity of FITC was measured with excitation at $485 \mathrm{~nm}$ and emission at $515 \mathrm{~nm}$ by fluorescence spectrophotometer (F-2500, HITACHI). The standard calibration curve of FITC was used to quantify and measure the concentration of FITC in $1 \mathrm{~mL}$ of FITC-PLGA NPs solution. From the derived concentration of FITC, the content of FITC in PLGA NPs can be calculated.

\subsection{Preparation of $P E G-\mathrm{NH}_{2}-Q D$-conjugated PLGA NPs $(Q D-P L G A N P S)$ and $P E G-N_{2}-Q D$-conjugated PLGA with FITC-encapsulated NPS (QD-FITC-PLGA NPs)}

Thirty microliters of PEG- $\mathrm{NH}_{2}-\mathrm{QD}(8 \mu \mathrm{M})$ and $1 \mathrm{~mL}$ of as-prepared PLGA NPs solution $(2.5 \mathrm{mg} / \mathrm{mL})$ were mixed in a $1.5 \mathrm{~mL}$ eppendorf. EDC $(0.1 \mathrm{mg})$ was added into the eppendorf and incubated for $1 \mathrm{~h}$. The mixture was centrifuged at $8000 \mathrm{rpm}$ for $5 \mathrm{~min}$ and the supernatant was discarded. The precipitates were washed twice using deionized water and the QDPLGA NPs were obtained after re-dispersing the precipitates in deionized water.

\subsection{Modification of NLS peptides on $Q D-P L G A$ NPs (NLS-QD-PLGA NPS)}

One hundred microliters of QD-PLGA NPs $(25 \mathrm{mg} / \mathrm{mL})$ and $20 \mu \mathrm{L}$ of SMCC solution (dissolved in DMSO) were mixed in a $1.5 \mathrm{~mL}$ eppendorf. The mixture was incubated for $4 \mathrm{~h}$ at $4{ }^{\circ} \mathrm{C}$ and centrifuged at $8000 \mathrm{rpm}$ for $5 \mathrm{~min}$. The precipitates were washed with $1 \mathrm{~mL}$ of deionized water before adding $10 \mu \mathrm{L}$ of deionized water. Seventy-five microliters of thiol-terminal modified NLS peptides $(10 \mu \mathrm{M})$ was added into SMCC-modified QD-PLGA 
NPs and incubated at $4{ }^{\circ} \mathrm{C}$ for $4 \mathrm{~h}$. After centrifugation at $8000 \mathrm{rpm}$ for $5 \mathrm{~min}$, the precipitates were re-dispersed in water to fabricate NLS-QD-PLGA NPs.

\subsection{Preparation of $\mathrm{F}_{3} \mathrm{O}_{4} \mathrm{Ns}$-conjugated PLGA $\mathrm{NPs}\left(\mathrm{F}_{3} \mathrm{O}_{4}-\mathrm{PLGA} \mathrm{NPS}\right)$}

Water-soluble $\mathrm{Fe}_{3} \mathrm{O}_{4} \mathrm{NPs}$ with $-\mathrm{NH}_{2}$ terminal groups were synthesized as previously reported [20]. Five milliliters of $2.5 \mathrm{mg} / \mathrm{mL}$ PLGA NPs solution was mixed with $20 \mu \mathrm{L}$ of $\mathrm{Fe}_{3} \mathrm{O}_{4}$ NPs $(0.2 \mathrm{~mm}$ nanoparticles) in a $20 \mathrm{~mL}$ of glass bottle. EDC $(0.2 \mathrm{mg})$ was added into the mixed solution and incubated for $1 \mathrm{~h}$. PLGA NPs were collected and excess $\mathrm{Fe}_{3} \mathrm{O}_{4}$ NPs were removed by centrifugation at $6000 \mathrm{rpm}$ for $5 \mathrm{~min}$. The precipitates were washed with deionized water twice. The collected $\mathrm{F}_{3} \mathrm{O}_{4}-\mathrm{PLGA}$ NPs were obtained after re-dispersion in deionized water. The $\mathrm{F}_{3} \mathrm{O}_{4}-$ PLGA NPs were in $\mathrm{HCl}$ aqueous $(6 \mathrm{~N})$ and the iron concentrations were measured by atomic absorption spectrometer (AA).

\subsection{Nanoparticle characterization}

Electron micrographs of various PLGA NPs were carried out by placing a drop of the sample onto a copper mesh coated with an amorphous carbon film and dried in a vacuum desiccator. The PLGA NPs were stained with phosphotunstic acid before the TEM images were taken. The mean diameter and morphology of PLGA NPs were characterized by TEM. The surface potential of PLGA NPs was determined by zeta potential (Zetasizer 3000HSAdvanced).

\subsection{In vitro release studies}

The FITC-PLGA NPs $(500 \mu \mathrm{L})$ was added directly into a $1.5 \mathrm{~mL}$ of eppendrof containing PBS buffer ( $1 \mathrm{~mL}, 10 \mathrm{~mm}, \mathrm{pH}$ 7.4). After specific time intervals, supernatant containing free FITC from FITC-PLGA NPs was collected by centrifugation at $13,000 \mathrm{rpm}$ for $10 \mathrm{~min}$. A control experiment was performed to determine the total amount of FITC in FITC-PLGA NPs by directly adding acetone to dissolve FITC-PLGA NPs and measuring the FITC in the supernatant. The percentage of FITC release at each time point was determined by fluorescence spectrophotometer and the fluorescent intensity ratio of FITC sample to the control were calculated at the emission wavelength of $515 \mathrm{~nm}$.

\subsection{Localization of PLGA nanoparticles in cell nuclei}

Human cervical cancer HeLa cells were cultured in DMEM (containing $10 \%$ fetal bovine serum, $1 \%$ penicillin) at $37{ }^{\circ} \mathrm{C}$ supplied with $5 \% \mathrm{CO}_{2} /$ $95 \%$ air. Cells were trypsinized and seeded in 4-well chamber slides with a density of $1 \times 10^{4}$ cells/well. After $24 \mathrm{~h}$ of incubation, each well was washed by PBS twice, followed by addition of $0.375 \mu \mathrm{g} / 500 \mu \mathrm{L}$ QD-PLGA NPs and NLS-QD-PLGA nanoparticles. The treated cells were incubated for 1, 3, 12, and $24 \mathrm{~h}$. The cells were then fixed by $4 \%$ paraformaldhyde/PBS for $1 \mathrm{~h}$ in room temperature for further laser confocal microscope analysis.

\subsection{Study of the uptake of nanoparticles by SD rat organs}

The rat brain, heart, lung, liver, kidney and spleen were obtained from 5 -day-old SD pups. The organs were incubated in 24-well plates and cultured in $1 \mathrm{~mL}$ conditioned medium. Twenty microliters of QD-PLGA NPs was added into each well. After incubation at $37{ }^{\circ} \mathrm{C}$ for $16 \mathrm{~h}$, the conditioned medium was removed and the organs were washed thrice with PBS. Wavelength-resolved spectral imaging was obtained using a spectral imaging system (Ivis 50 imager). The excitation was $445-490 \mathrm{~nm}$ and the emission filter was $515-575 \mathrm{~nm}$

\subsection{Characterization of the $r 2$ relaxivities}

These experiments were performed using spectroscopy (3 T MRI Biospec; Bruker, Ettlingen, Germany). A gradient system was mounted on the table of the $3 \mathrm{~T}$ magnet with an inner diameter of $6 \mathrm{~cm}$ and a maximal gradient strength of $1000 \mathrm{mT} / \mathrm{m}$ was used to yield high-resolution images. A quadrature coil with an inner diameter of $3.5 \mathrm{~cm}$ was used for $\mathrm{RF}$ transmission and reception. For in vitro $\mathrm{MR}$ images and $\mathrm{T}_{2}$ measurements, $\mathrm{Fe}_{3} \mathrm{O}_{4}$ and $\mathrm{F}_{3} \mathrm{O}_{4}-\mathrm{PLGA}$ NPs were dispersed in various iron ion concentrations $(0.0046,0.0092,0.046$, $0.092,0.46,0.92$ and $4.6 \mathrm{~mm})$. The array was embedded in a phantom to allow the appropriate image acquisition. Acquired images had a matrix size of $256 \times 192 \mathrm{~mm}$, a field of view of $60 \times 60 \mathrm{~mm}$, and a slice thickness of $3 \mathrm{~mm}$ yielding an in-plane resolution of $234 \mu \mathrm{m}$ after image smoothing. Both $\mathrm{T}_{1}$ - and $\mathrm{T}_{2}$-weighted images were acquired using a multi-slice multi-echo ( $\mathrm{T}_{1}$-weighted) and fast spin echo $\left(\mathrm{T}_{2}\right.$-weighted) sequence with a repetitiontime/echo-time (TR/TE) of 472/9.4 ms with a number of averages (NEX) of 8 and TR/TE of $4500 / 65 \mathrm{~ms}$ with a NEX of 6 , respectively. $T_{1}$ values were measured using a multi-slice multi-echo sequence with a repetition-time (TR) of $6000 \mathrm{~ms}$, an echo time (TE) of $8.7 \mathrm{~ms}$, and 45 inversion recovery points (TI from 13.3 to $6000 \mathrm{~ms}$ ). The field of view was $60 \times 60 \mathrm{~mm}$, the slice thickness was $6 \mathrm{~mm}$, and the image matrix was $128 \times 128$. This allowed for simultaneous imaging of 26 vials with $0.3 \mathrm{~mL}$ of contrast agent for each vial. An average signal of 50 voxels was evaluated for all TI values. $T_{2}$ values were performed with a spin echo sequence of TR/TE of 4000/10.1 ms, 60 echo points of 60 , and a NEX of 5 . The field of view was $60 \times 60 \mathrm{~mm}$, the slice thickness was $6 \mathrm{~mm}$, and the imaging plane was $256 \times 192$.

\section{Results and discussion}

In this study, we have successfully used nanoprecipitation method to prepare non-stabilizer-coated PLGA NPs of three different sizes with small size distributions. The mean diameters of PLGA NPs were $72 \pm 6.8 \mathrm{~nm}, 125.8 \pm 6.8 \mathrm{~nm}$ and $192.6 \pm 15.2 \mathrm{~nm}$ (Fig. 1a-c). During our nanoparticle formation, acetone/ethanol aqueous was used as the solvent/nonsolvent pair, and ethanol, instead of PVA, was used as the stabilizer to avoid PLGA NP aggregations. Because ethanol has a similar unit structure to PVA, it can produce similar interaction as shown between PVA and PLGA NPs [21]. Acetone and ethanol can be completely evaporated from solventevaporation process after nanoparticle formation. The yields of PLGA NPs were over $90 \%$ in all sizes. The size difference was controlled by either changing the concentration of PLGA in acetone or the molecular weight of PLGA used. For examples, $72 \pm 6.8 \mathrm{~nm}$ and $125.8 \pm 6.8 \mathrm{~nm}$ of PLGA NPs were prepared from $10 \mathrm{mg} / \mathrm{mL}$ and $20 \mathrm{mg} / \mathrm{mL}$ of PLGA (M.W.: 35,000-65,000 Da) in acetone, respectively. PLGA NPs of $192.6 \pm 15.2 \mathrm{~nm}$ were synthesized using the same concentration as $72 \pm 6.8 \mathrm{~nm}$ PLGA NPs but using PLGAs with a smaller molecular weight (M.W.: 16,000-35,000 Da) as its precursor. The prepared PLGA NPs were stable for over one month at room temperature.

To demonstrate the modification of functional substrates on the surface of non-stabilizer-coated PLGA NPs, $\mathrm{PEG}-\mathrm{NH}_{2}$ modified quantum dots ( $\mathrm{PEG}-\mathrm{NH}_{2}-\mathrm{QD}$ ) were used to conjugate on the COOH-terminated groups of PLGA NPs by adding 1-ethyl-3(3-dimethylaminopropyl)-carbodiimide (EDC) (Fig. 1d). It took $2 \mathrm{~h}$ to conjugate PLGA NPs with biotin-(poly(ethylene glycol))amine (BPEG) with our conjugation method whereas it took at least $24 \mathrm{~h}$ for other groups [19]. According to the result of zeta potential, the surface charge of PLGA NPs changed from -14.92 to $+22.71 \mathrm{mV}$ after conjugation of QD. In Fig. 1d, PEG- $\mathrm{NH}_{2}-\mathrm{QD}$ coated PLGA NPs (QDPLGA nanoparticles) showed a larger size $(\sim 168 \mathrm{~nm})$ 

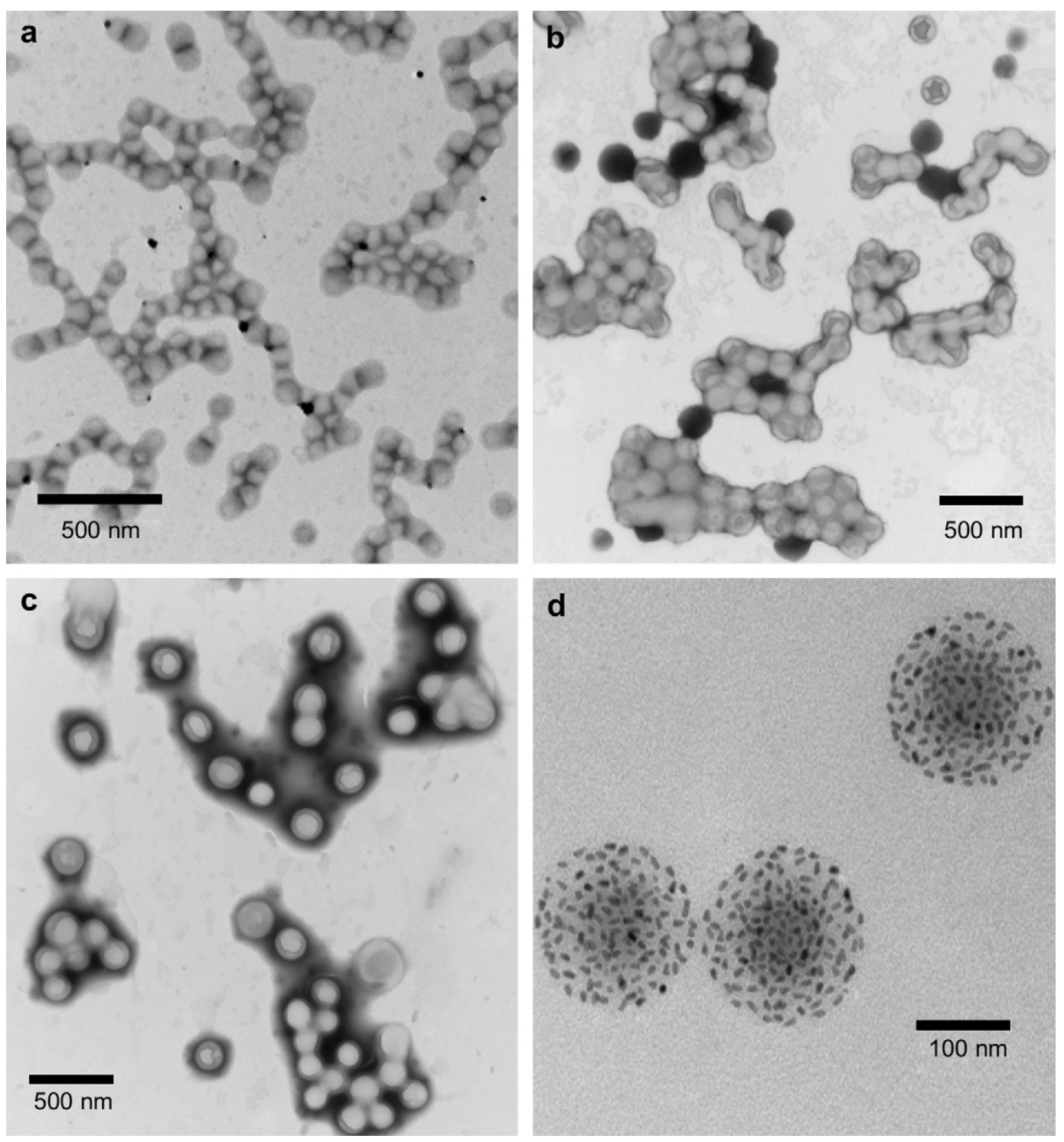

Fig. 1. The TEM images of (a) $72 \pm 6.8 \mathrm{~nm}$ PLGA NPs, (b) $125.8 \pm 6.8 \mathrm{~nm}$ PLGA NPs, (c) $192.6 \pm 15.2 \mathrm{~nm}$ PLGA NPs and (d) QD-PLGA NPs. (a) -(c) were stained with $2 \%$ phosphotunstic acid for TEM measurements.

comparing with the original PLGA nanoparticles $(72 \mathrm{~nm})$. The diameter of PEG- $\mathrm{NH}_{2}-\mathrm{QD}$ was approximately $12.1 \pm$ $1.2 \mathrm{~nm}$ (see Supporting Information, Fig. S1). The increase of size might be due to the repulsion effect caused by the steric hindrance between the $\mathrm{PEG}-\mathrm{NH}_{2}-\mathrm{QDs}$ on the surface. To reduce the repulsion effect, the distance between the $\mathrm{PEG}-\mathrm{NH}_{2}-\mathrm{QDs}$ increased hence the size increased.

To examine the encapsulation capability of PLGA NPs, fluorescein isothiocyanate (FITC) was entrapped inside PLGA NPs during nanoprecipitation of PLGA. The efficiency of FITC entrapment was approximately $90 \%$. The amount of FITC in $72 \mathrm{~nm}$ of PLGA NPs $(\% \mathrm{w} / \mathrm{w})$ was about $0.2 \%$. The color of FITC-encapsulated PLGA NPs (FITC-PLGA NPs) was light yellow in aqueous and can be observed with naked eyes. The FITC release from PLGA NPs dissolved in phosphate buffer saline (PBS, pH 7.4) was shown in Fig. 2. The release exerted a two-phase pattern - an initial burst release

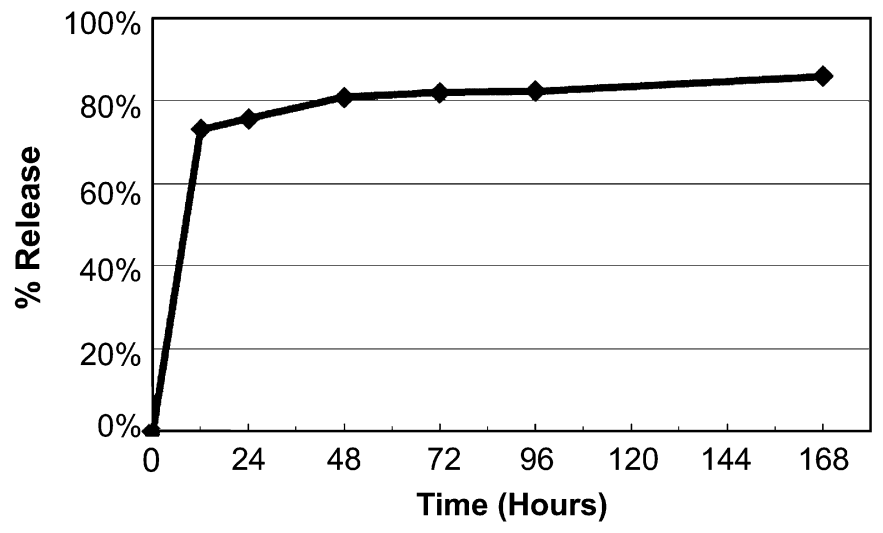

Fig. 2. In vitro release profile of FITC from FITC-PLGA NPs in PBS (pH 7.4) (FITC content $=0.2 \% \mathrm{w} / \mathrm{w})$. 
and a delayed slow release. The initial exponential profile showed a fast release for up to $68 \%$ within $3 \mathrm{~h}$ (see Supporting Information, Fig. S2) and was close to $80 \%$ within $24 \mathrm{~h}$, followed by a slow release gradually increased for up to $86 \%$ of FITC after $168 \mathrm{~h}$. The rapid release phase was probably due to adsorbed FITC near the surface of nanoparticles which readily diffused into the medium. The slow release of FITC may come from the release during PLGA degradation. Since the drug release from PLGA NPs may depend on three primary mechanisms - swelling, diffusion, and degradation [12], both the size of the loaded drug and the diameter of PLGA NPs impact the release profile. For example, Noemi et al. and Lee et al. studied paclitaxel release from PLGA NPs and found that it took 60 days to release $80 \%$ of the loaded paclitaxel $[22,23]$. By contrast, in other studies using smaller procaine hydrochloride or indocyanine green (ICG) molecules, it took only $15 \mathrm{~min}$ or $8 \mathrm{~h}$ to release $80 \%$ of the molecules from PLGA NPs with the size of $100-200 \mathrm{~nm}$ or $300-400 \mathrm{~nm}$, respectively $[9,16]$. In our studies, swelling of smaller PLGA NPs $(72 \mathrm{~nm})$ took place fast after being exposed to the medium which led to rapid release of FITC within $3 \mathrm{~h}$.

To fabricate multifunctional imaging probes using PLGA NPs, we entrapped FITC (emission at $515 \mathrm{~nm}$ ) inside 72 nm-sized PLGA NPs which were conjugated with PEG$\mathrm{NH}_{2}-\mathrm{QD}$ (emission at $655 \mathrm{~nm}$ ) (QD-FITC-PLGA NPs). Therefore, the composites QD-FITC-PLGA NPs can exert two different emission wavelengths simultaneously using the same excitation energy at $488 \mathrm{~nm}$ as shown in Fig. 3. As expected, the emission intensity of FITC (Fig. 3a) was weaker as compared with that of $\mathrm{PEG}-\mathrm{NH}_{2}-\mathrm{QD}$ (Fig. 3b). These results demonstrate the potential of PLGA NPs for serving as a bimodal imaging probe.

The substrate-encapsulated delivery system of PLGA NPs has been widely used to deliver drug or DNA into cells $[2,3,8,11]$. In many cases, it is important that the substances carried are delivered not only to the cytoplasm but also into the nucleus where they perform their functions. For gene therapy, it is important that the genes carried are delivered into the nucleus for DNA integration. For cancer treatment, drugs may also be delivered into cancer cell nuclei to induce apoptosis. In this study, we carried out to test PLGA NP's ability to enter the nucleus by incorporating nuclear localization signal (NLS) peptides which have been shown to facilitate nuclear targeting of DNA [24]. The QD-PLGA NPs (emission at $525 \mathrm{~nm}$ ) were conjugated with thiol-terminal NLS peptides using succinimidyl-4-[N-maleimidomethyl]-cyclohexane-1carboxylate (SMCC) (see experimental details in Supporting Information). The resulting NLS-QD-PLGA NPs were tested for HeLa cell uptake in culture. Small PLGA NPs (72 nm), adv-NLS and sv40-NLS were used for control. The adv-NLS peptides (from adenovirus fiber protein, sequence: CGGGPKKKRKVGG) exert higher nuclear entrance efficiency compared with sv40-NLS peptides (from the large T antigen of SV-40 virus, sequence: CGGFSTSLRARKA) which have lower or even no nuclear entrance (Fig. 4) [25,26]. As shown in Fig. 4, QD-PLGA NPs had a lower uptake efficiency than that of adv-NLS-QD-PLGA and sv40-NLS-QD-PLGA NPs at three different time points tested, 3, 12 and $24 \mathrm{~h}($ Fig. $4 \mathrm{a}-\mathrm{c})$. Furthermore, the NLSPLGA NPs were readily uptaken by HeLa cells into both the cytoplasm and the nucleus whereas the sv40-NLS-PLGA NPs were only uptaken into the cytoplasm but not the nucleus (Fig. $4 \mathrm{~d}-\mathrm{f}$ ) as compared with adv-NLS-PLGA NPs entering nuclei (Fig. $4 \mathrm{~g}-\mathrm{i}$ ). We also observed that at $3 \mathrm{~h}$, more advNLS-PLGA NPs were uptaken by cells than QD-PLGA NPs or sv40-NLS-QD-PLGA NPs (Fig. 4a,d,g). It should be noted that the size of nuclear pore complexes is normally between 20 and $50 \mathrm{~nm}[27,28]$ and therefore becomes a limitation for using $72 \mathrm{~nm}$ PLGA nanoparticles. The final size of PLGA NPs after conjugation with QDs made the entrance into the nucleus even more difficult. It was believed that the entry of adv-NLS-PLGA NPs into the nucleus was enabled by its degradable characteristic. Once the adv-NLS-PLGA
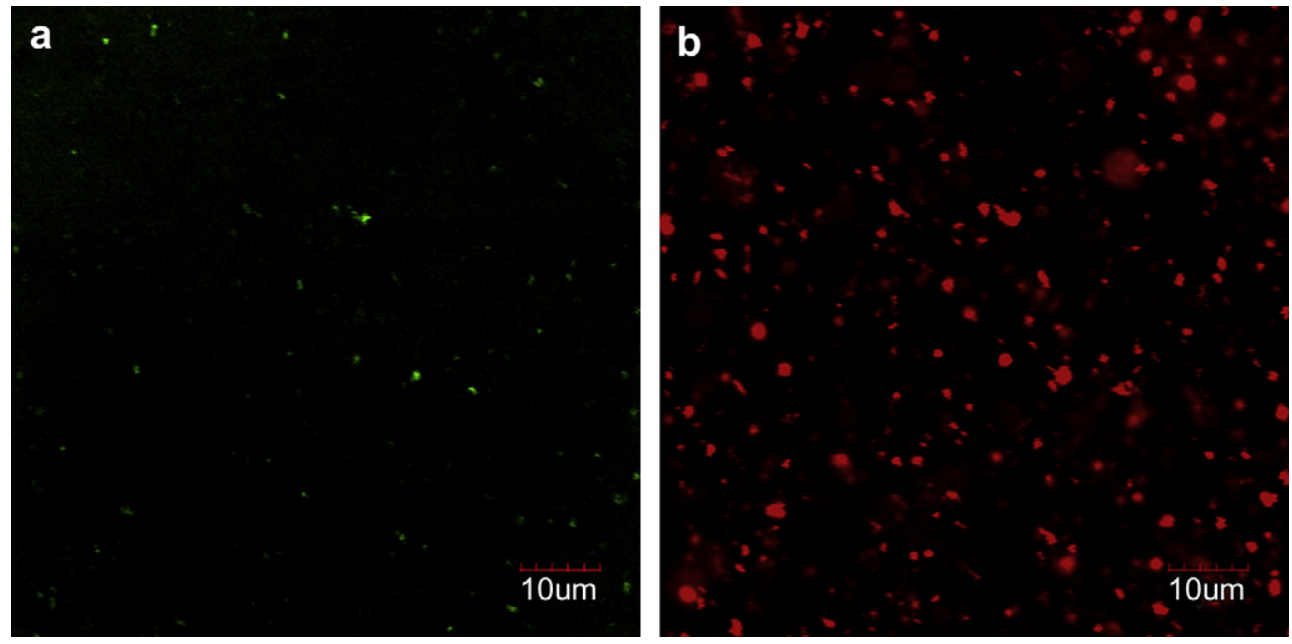

Fig. 3. High-resolution fluorescence images of QD-FITC-PLGA NPs. The excitation of QD-FITC-PLGA NPs was at $488 \mathrm{~nm}$ and the emission wavelength was at $515 \mathrm{~nm}$ for FITC (a) or $655 \mathrm{~nm}$ for PEG- $\mathrm{NH}_{2}-\mathrm{QD}$ (b). 
$3 \mathrm{~h}$
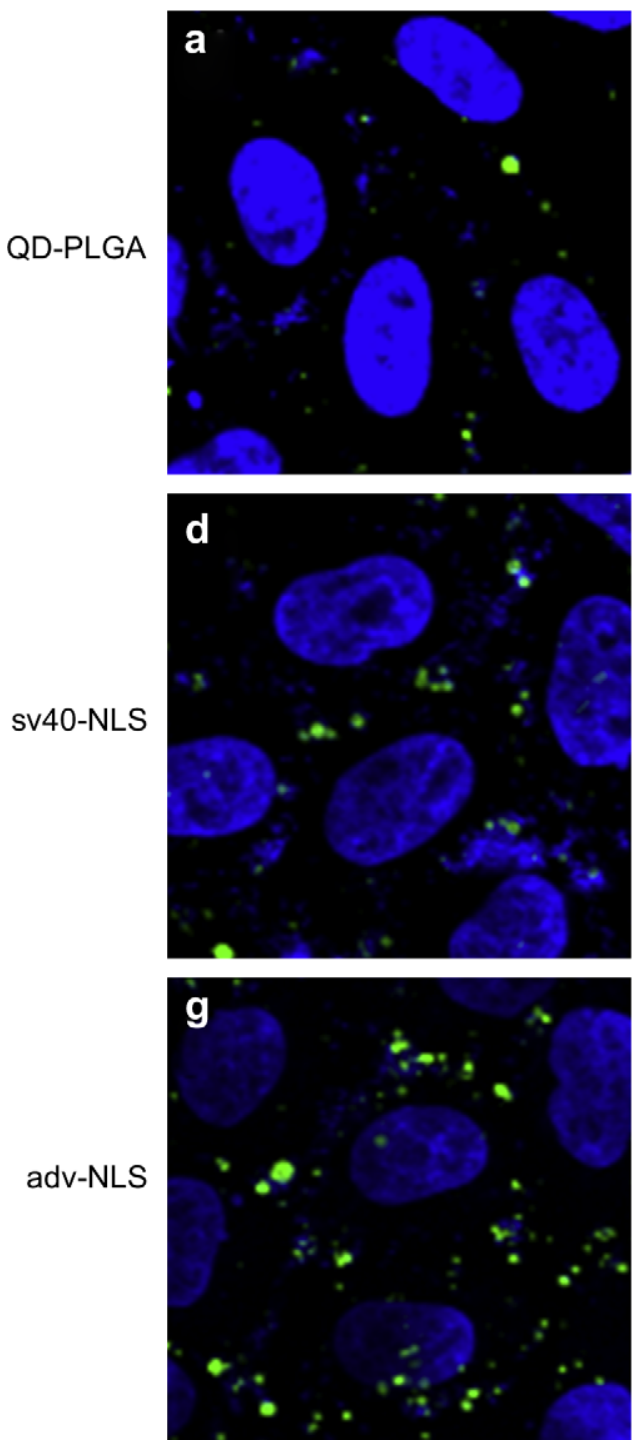

$12 \mathrm{~h}$
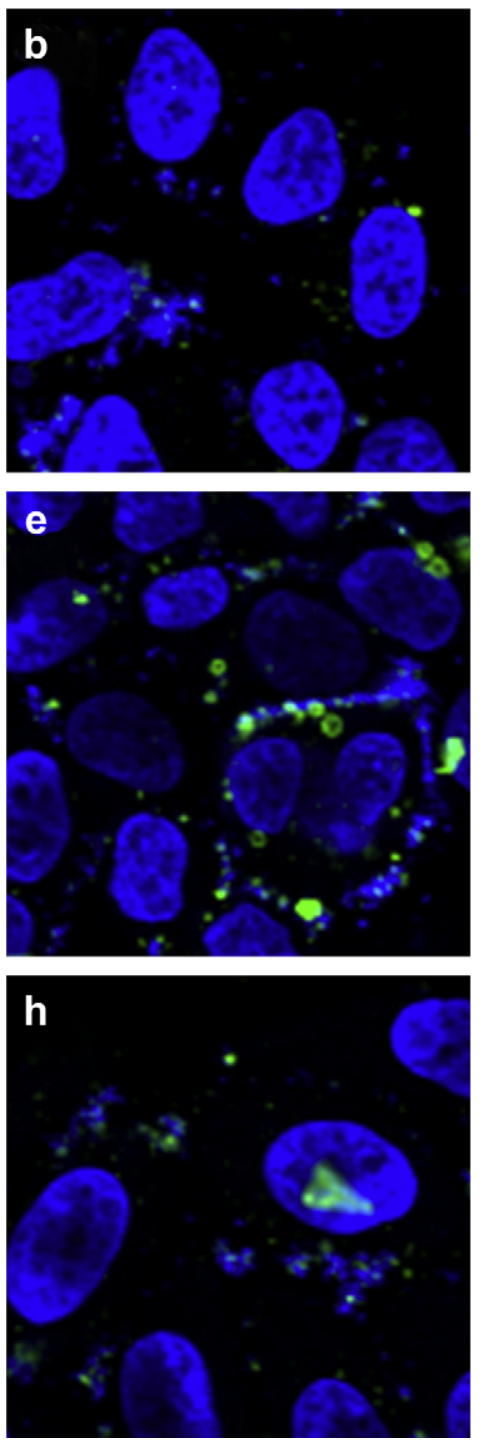

$24 \mathrm{~h}$
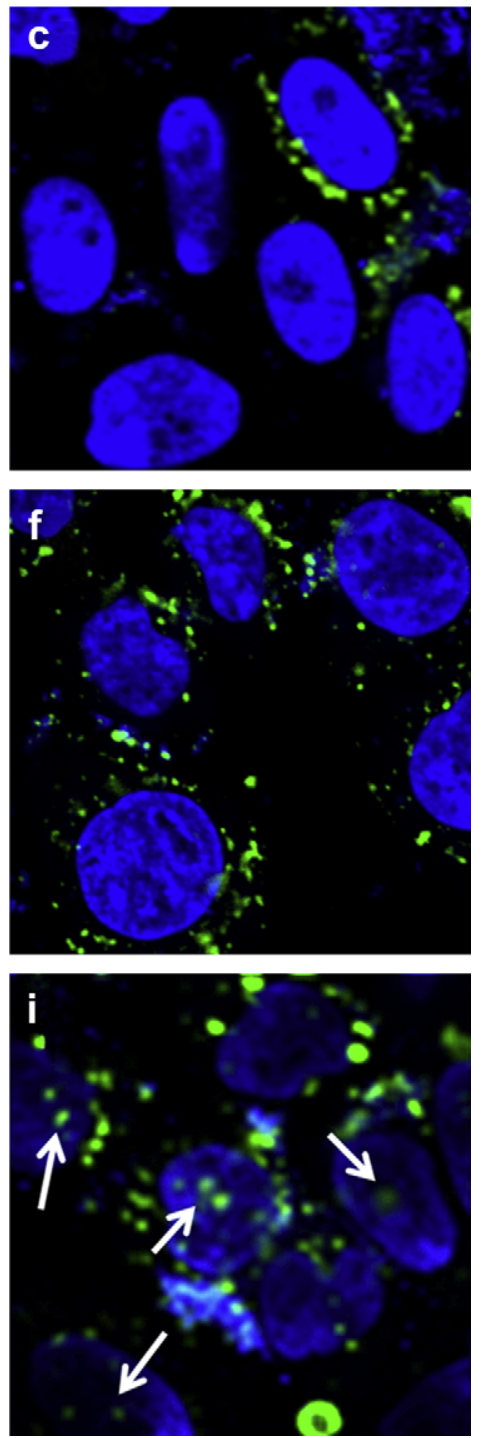

Fig. 4. High-resolution fluorescence images of PLGA NP nuclear translocation using laser confocal microscopy (excitation: $488 \mathrm{~nm}$ ). HeLa cells were incubated with QD-PLGA NPs, sv40-NLS-QD-PLGA NPs, and adv-NLS-QD-PLGA NPs for 3, 12, and $24 \mathrm{~h}$, respectively. Blue and green colors represent cell nucleus (stained with DAPI) and QD-PLGA NPs, respectively. The arrows in Fig. 4i indicate adv-NLS-PLGA NPs localized in the nuclei.

NPs degrade to a size smaller than the pore size, they can penetrate into the nucleus more easily. Since the pore size between the cytoplasm and nucleus is still unclear, it is equally possible that it may be bigger than $50 \mathrm{~nm}$.

Tissue uptake of PLGA NPs can provide important information for in vivo applications such as for drug delivery, gene transfer, and magnetic resonance imaging (MRI) imaging. To investigate the impact of different sizes of PLGA NPs on the uptake by tissue, QD-PLGA NPs with three different diameters of PLGA NPs were used. We found that QD-PLGA NPs were uptaken by several tissues after $16 \mathrm{~h}$ in culture. The brain had the best uptake among all tissues tested whereas uptake in the spleen was not detected (Fig. 5). As expected, certain degree of uptakes was observed in the liver, kidney, heart and lung, with a decrease of intensity detected. It may be reasonable to observe more uptake in the brain tissue than in the others due to the absence of bloodbrain-barrier (BBB), a membrane structure that acts primarily to protect the brain from foreign materials or organisms in the blood. More QD-PLGA NPs uptakes were observed in the brain, liver and kidney after prolonged incubation time. However, in tissues such as lung and spleen where low or no uptake was observed initially, there was still no improvement after extending the incubation time (see Supporting Information, Fig. S3). Interestingly, we found no difference of uptake using different sizes of PLGA NPs, implying that tissue factor is the major determinant for uptake (Fig. 5 and Supporting Information, Figs. S3 and S4).

We next explored the possibility of using PLGA NPs combined with iron oxide for MRI imaging, which was traditionally performed through hydrophobic oleic acid coating on the surface of iron oxide NPs $[29,30]$. To encapsulate hydrophobic 

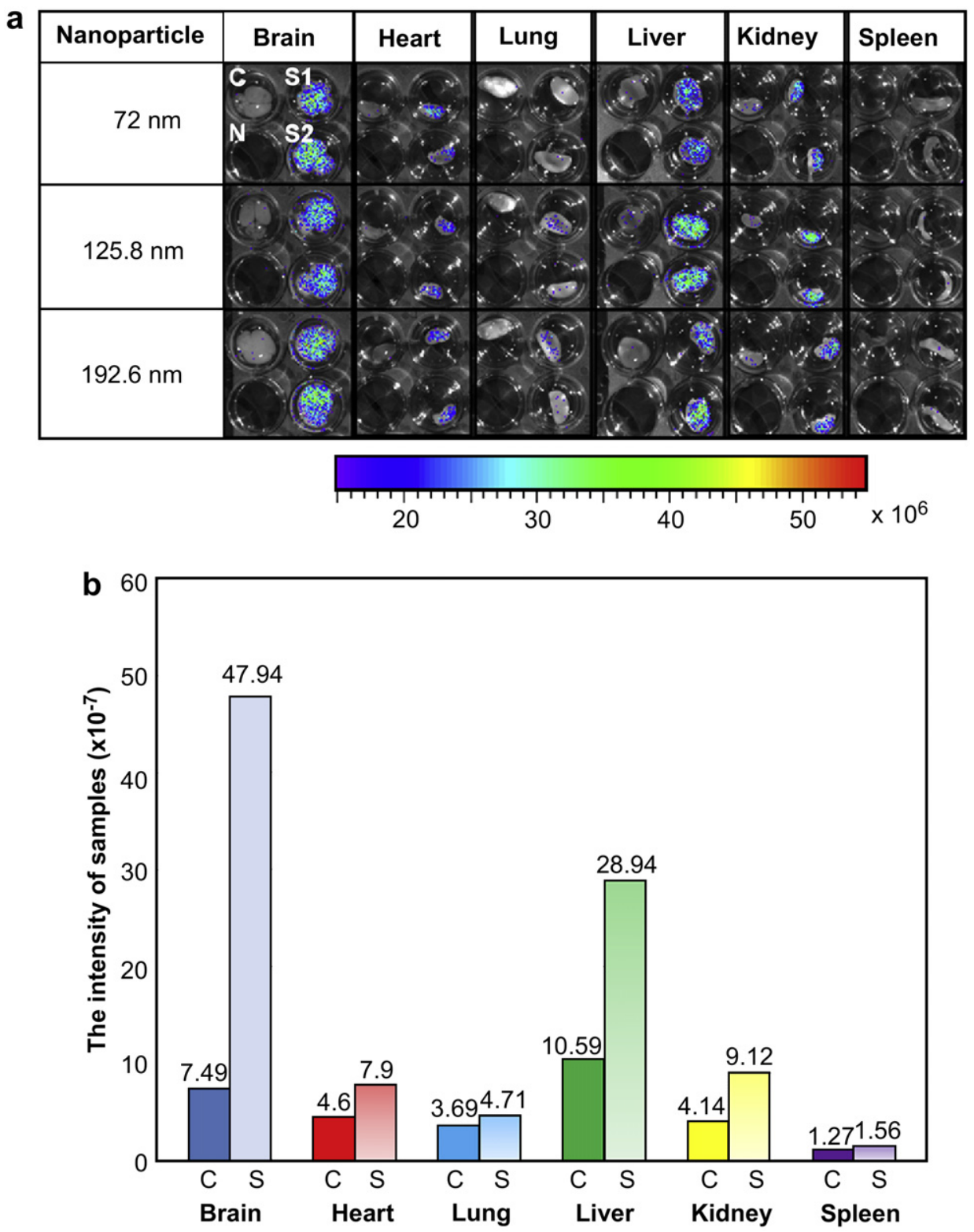

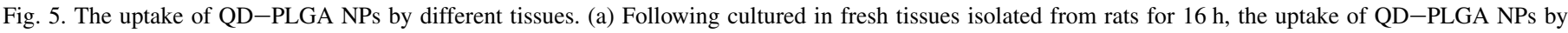

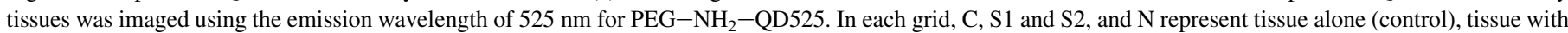

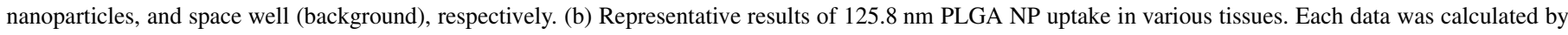
three repeats of experiments with the average of intensities in S1 and S2 (S) minus the background signal.

magnetic nanoparticles into PLGA, a combined emulsification-diffusion, double emulsion and emulsion-evaporation methods were employed to prepare the iron oxide/PLGA composites. Although these iron oxide-encapsulated PLGA NPs could be used as contrast agents for MRI, the oleic acid-coated iron oxides were not an ideal material for living organisms because of toxicity. To avoid this, water-soluble non-cytotoxic $\mathrm{Fe}_{3} \mathrm{O}_{4}$ NPs were conjugated on the PLGA NP surface using 1-ethyl-3-(3-dimethylaminopropyl)-carbodiimide (EDC) as conjugating agent. We have previously synthesized $\mathrm{Fe}_{3} \mathrm{O}_{4}$ NPs $(6.2 \pm 2.1 \mathrm{~nm})$ with $-\mathrm{NH}_{2}$ groups exposed on the surfaces [20], the same approach which we used here again to fabricate $\mathrm{Fe}_{3} \mathrm{O}_{4}$-conjugated PLGA NPs $\left(\mathrm{Fe}_{3} \mathrm{O}_{4}-\mathrm{PLGA}\right.$ NPs) (see Supporting Information, Fig. S5). To the best of our knowledge, this is the first approach to conjugate biocompatible functional $\mathrm{Fe}_{3} \mathrm{O}_{4}$ NPs on the surface of PLGA NPs. Fig. 6 shows the MR images $\left(\mathrm{T}_{2}\right.$ weight) of $\mathrm{Fe}_{3} \mathrm{O}_{4}-\mathrm{PLGA}$ NPs with 72 and $192 \mathrm{~nm}$ PLGA NPs and of bare $\mathrm{Fe}_{3} \mathrm{O}_{4}$ nanoparticles. As demonstrated, the $\mathrm{Fe}_{3} \mathrm{O}_{4}-\mathrm{PLGA}$ NPs have a higher contrast enhancement than that of bare $\mathrm{Fe}_{3} \mathrm{O}_{4}$ nanoparticles in $\mathrm{T}_{2}$-weighted imaging. We also found that $\mathrm{Fe}_{3} \mathrm{O}_{4}$-PLGA NPs in larger size exhibited more contrast effect than the smaller ones did. All three types of NPS showed MR imaging in a dose-dependent manner (Fig. 6). Interestingly, we also observed that $\mathrm{Fe}_{3} \mathrm{O}_{4}-$ PLGA NPs made contrast darkening at lower iron ion concentration compared with bare $\mathrm{Fe}_{3} \mathrm{O}_{4}$ NPs. We therefore measured the proton relaxivities $r 2$ of the bare $\mathrm{Fe}_{3} \mathrm{O}_{4}$ and $\mathrm{Fe}_{3} \mathrm{O}_{4}$-PLGA NPs by determining 


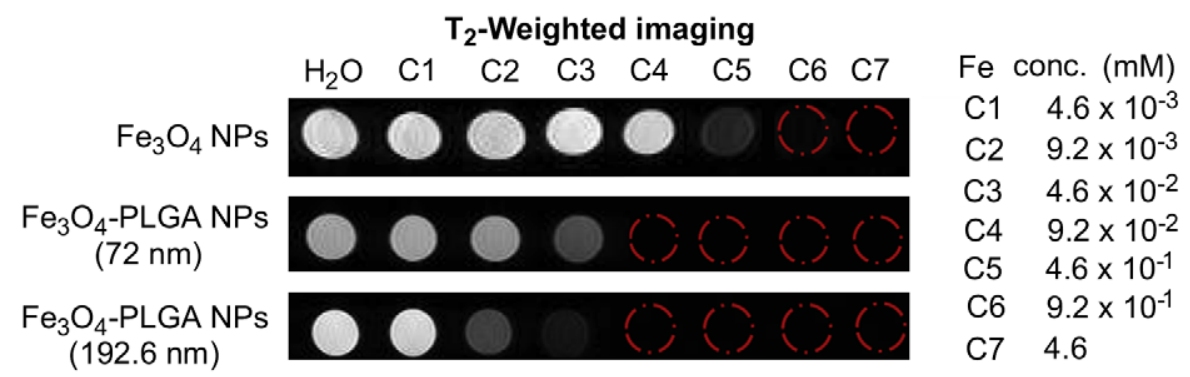

Fig. 6. MRI images of $\mathrm{Fe}_{3} \mathrm{O}_{4}-$ PLGA NPs. Shown are representative images acquired from $\mathrm{T}_{2}$-weighted images using an array of concentration gradient of Fe $\mathrm{O}_{4}$ NPs and $\mathrm{Fe}_{3} \mathrm{O}_{4}-$ PLGA NPs in two different sizes.

the transverse $\left(\mathrm{T}_{2}^{-1}\right)$ relaxation rates at various concentrations using a 3 T MR system (see Supporting Information, Fig. S6). The $r 2$ relaxivity of the bare $\mathrm{Fe}_{3} \mathrm{O}_{4}$ nanoparticles and $\mathrm{Fe}_{3} \mathrm{O}_{4}$ PLGA NPs with 72 and $192 \mathrm{~nm}$ PLGA NPs were 84.7, 294.2, and $817.6 / \mathrm{s} / \mathrm{mm}$, respectively. $\mathrm{Fe}_{3} \mathrm{O}_{4}-$ PLGA NPs in the size of $192 \mathrm{~nm}$ had higher iron oxide accumulation on the surface $\left(3 \times 10^{16} \mathrm{Fe}_{3} \mathrm{O}_{4} \mathrm{NPs} / \mathrm{mg}\right.$ PLGA) compared with that using $\mathrm{Fe}_{3} \mathrm{O}_{4}-$ PLGA NPs with the size of $72 \mathrm{~nm}$ PLGA $\left(8 \times 10^{12}\right.$ $\mathrm{Fe}_{3} \mathrm{O}_{4} \mathrm{NPs} / \mathrm{mg}$ PLGA). Therefore the more $\mathrm{Fe}_{3} \mathrm{O}_{4}$ nanoparticles on the PLGA, the higher the transverse relaxation rate $\left(\mathrm{T}_{2}^{-1}\right)$ and thus the higher degree of heterogeneity of magnetic field which in turn produced phantom images for $\mathrm{T}_{2}$-weighted imaging.

\section{Conclusion}

We have successfully synthesized PLGA NPs with three different sizes and demonstrated their potential applications for biomedical use. The encapsulation of FITC and conjugation of QDs with the PLGA NPs as well as their controllable release kinetics allow us to apply these NPs for molecular imaging and drug delivery. Furthermore, we demonstrate that NLS-QD-PLGA NPs may travel into the nucleus, a potential approach for gene targeting. Finally, we show evidence that the composite of $\mathrm{Fe}_{3} \mathrm{O}_{4}$ and PLGA NPs exhibits a high $r 2$ relaxivity and can thus be used as a powerful MRI imaging system. Future work could provide important insights into the applications in vivo.

\section{Acknowledgements}

We would like to thank the National Science Council of Taiwan for financially supporting this work.

\section{Appendix. Supplementary material}

TEM images of PEG- $\mathrm{NH}_{2}-$ QDs and $\mathrm{Fe}_{3} \mathrm{O}_{4}-$ PLGA NPs, in vitro release profile of FITC, the time effect of QD-PLGA NPs in tissues, the quantification of uptake efficiency of PLGA in various tissues, and the $r 2$ relaxivity curves of $\mathrm{Fe}_{3} \mathrm{O}_{4}-\mathrm{PLGA}$ NPs. Supplementary material associated with this article can be found at doi: 10.1016/j.biomaterials.2008.01.010.

\section{References}

[1] Shaoo SK, Panda AK, Labhasetwar V. Characterization of porous PLGA/ PLA microparticles as a scaffold for three dimensional growth of breast cancer cells. Biomacromolecules 2005;6:1132-9.

[2] Jain RA. The manufacturing techniques of various drug loaded biodegradable poly(D,L-lactide-co-glycolide acid) (PLGA) devices. Biomaterials 2000;21:2475-90.

[3] Prabha S, Labhasetwar V. Nanoparticle-mediated wild-type p53 gene delivery results in sustained antiproliferative activity in breast cancer cells. Mol Pharm 2004;1:211-9.

[4] Gvili K, Benny O, Danino D, Machluf M, Labhasetwar V. Poly(D,L-lactideco-glycolide acid) nanoparticles for DNA delivery: waiving preparation complexity and increasing efficiency. Biopolymers 2007;85:379-91.

[5] Na K, Kim S, Park K, Kim K, Woo DG, Kwon IC, et al. Heparin/ poly(L-lysine) nanoparticle-coated polymeric microspheres for stem-cell therapy. J Am Chem Soc 2007;129:5788-9.

[6] Ugo B, Eric A, Eric D. Development of a nanoprecipitation method intended for the entrapment of hydrophilic drugs into nanoparticles. Eur J Pharm Sci 2005;24:67-75.

[7] Yvette NK, Radovan C, Joselyne F, Myriam B, Robert G, Eric A. Preparation and characterization of sterile sub- $200 \mathrm{~nm}$ meso-tetra(4hydroxyphenyl)porphyrin-loaded nanoparticles for photodynamic therapy. Eur J Pharm Biopharm 2003;55:115-24.

[8] Cristina F, Sergio S, Rogerio G. Paclitxel-loaded PLGA nanoparticles: preparation, physicochemical characterization and in vivo anti-tumoral activity. J Control Release 2002;83:273-86.

[9] Vishal S, Mostafa S, Jun S. Indocyanine green-loaded biodegradable nanoparticles: preparation, physicochemical characterization and in vitro release. Int J Pharm 2004;278:293-301.

[10] Kim SH, Jeong JH, Chun KW, Park TG. Target-specific cellular uptake of PLGA nanoparticles coated with poly(L-lysine)-poly(ethylene glycol)-folate conjugate. Langmuir 2005;21:8852-7.

[11] Sahoo SK, Labhasetwar V. Enhanced antiproliferative activity of transferrin-conjugated paclitaxel-loaded nanoparticles is mediated via sustained intracellular drug retention. Mol Pharm 2005;2:373-83.

[12] Song KC, Lee HS, Choung IY, Cho KI, Ahn Y, Choi EJ. The effect type of organic phase solvents on the particle size of poly(D,L-lactide-coglycolide) nanoparticles. Colloids Surf A Physicochem Eng Asp 2006:276:162-7.

[13] Carlos EA, Challa SSK, Cristina MS. Size control of poly(D,L-lactide-coglycolide) and poly(D,L-lactide-co-glycolide)-magnetite nanoparticles synthesized by emulsion evaporation technique. Colloids Surf A Physicochem Eng Asp 2007;299:209-16.

[14] Zhang H, Cui W, Bei J, Wang S. Preparation of poly(D,L-lactide-coglycolide-co-caprolactone) nanoparticles and their degradation behavior in aqueous solution. Polym Degrad Stab 2006;91:1929-36.

[15] Lee SJ, Jeong JR, Shin SC, Kim JC, Chang YH, Lee KH, et al. Magnetic enhancement of iron oxide nanoparticles encapsulated with poly(D,L-lactide-co-glycolide). Colloids Surf A Physicochem Eng Asp 2005;255:19-25. 
[16] Thirumala G, Snjezana S, Martin CG, Lisbeth I, Davis SD. PLGA nanoparticles prepared by nanoprecipitation: drug loading and release studies of a water soluble drug. J Control Release 1999;57:171-85.

[17] Quintanar GD, Allemann E, Fessi H, Doclker E. Preparation techniques and mechanism of formation of biodegradable nanoparticles from preformed polymers. Drug Dev Ind Pharm 1998;24:1113-28.

[18] Chun KW, Yoo HS, Yoon JJ, Park TG. Biodegradable PLGA microcarriers for injectable delivery of chondrocytes: effect of surface modification on cell attachment and function. Biotechnol Prog 2004;20:1797-801.

[19] Weiss B, Schneider M, Muys L, Taetz S, Neumann D, Scharfer UF, et al. Coupling of biotin-(poly(ethylene glycol))amine to poly(D,L-lactide-coglycolide) nanoparticles for versatile surface modification. Bioconjug Chem 2007;18:1087-94.

[20] Shieh DB, Cheng FY, Su CH, Yeh CS, Wu MT, Wu YN, et al. Aqueous dispersions of magnetite nanoparticles with $\mathrm{NH}_{3}^{+}$surfaces for magnetic manipulations of biomolecules and MRI contrast agents. Biomaterials 2005;26:7183-91.

[21] Murakami H, Kobayashi M, Takeuchi H, Kawashima Y. Preparation of poly(D,L-lactide-co-glycolide) nanoparticles by modified spontaneous emulsification solvent diffusion method. Int. J. Pharm 1999;187: 143-52.

[22] Noemi C, Pilar C, Alejandro S, Fernando D, Maria AJ. PLGA:poloxamer and PLGA:poloxamine blend nanoparticles: new carriers for gene delivery. Biomacromolecules 2005;6:271-8.
[23] Lee KE, Kim BK, Yuk SH. Biodegradable polymeric nanospheres formed by temperature-induced phase transition. Biomacromolecules 2002;3:1115-9.

[24] Munkonge FM, Dean DA, Heillery E, Griesenbach U, Alton EW. Emerging significance of plasmid DNA nuclear import in gene therapy. Adv Drug Deliv Rev 2003;55:749-60.

[25] Tkachenko AG, Xie H, Liu Y, Coleman D, Glomm WR, Ryan J, et al. Multifunctional gold nanoparticle-peptide complexes for nuclear targeting. J Am Chem Soc 2003;125:4700-1.

[26] Tkachenko AG, Xie H, Liu Y, Coleman D, Glomm WR, Shipton MK, et al. Cellular trajectories of peptide-modified gold particle complexes: comparison of nuclear localization signals and peptide transduction domains. Bioconjug Chem 2004;15:482-90.

[27] Fahrenkrog B, Aebi U. The nuclear pore complex: nucleocytoplasmic transport and beyond. Nat Rev Mol Cell Biol 2003;4:757-66.

[28] Lechardeur D, Verkman AS, Lukacs GL. Intracellular routing of plasmid DNA during non-viral gene transfer. Adv Drug Deliv Rev 2005;57:755-67.

[29] Okassa LN, Marchais H, Eyrolles LD, Jonathan SC, Souce M, Dubois P, et al. Development and characterization of sub-micron poly(D,L-lactideco-glycolide) particles loaded with magnetite/maghhemite nanoparticles. Int J Pharm 2005;302:187-96.

[30] Lee SJ, Jeong JR, Shin SC, Kim JC, Chang YH, Chang YM, et al. Nanoparticles of magnetic ferric oxides encapsulated with poly(D,Llactide-co-glycolide) and their applications to magnetic resonance imaging contrast agent. J Magn Magn Mater 2004;272:2432-3. 